Acta Poetica $26(1-2)$

PRIMAVERA-OTOÑO

2005

\title{
La cosmología poética en los huaynos quechuas tradicionales
}

\author{
Martin Lienhard
}

\begin{abstract}
Las investigaciones recientes sobre las prácticas simbólicas andinas han demostrado que la expresión de lo "cosmológico" no es exclusiva de ninguno de los numerosos discursos (paralelos, superpuestos o imbricados) que componen, en los Andes centrales, una performance ritual y/o musical. Esta concepción de lo cosmológico se incrusta, también, en el discurso narrativo (códigos dramáticos y verbales) que las prácticas rituales, los cantos, entre otros, actualizan. En este trabajo estudio la "cosmología poética" que subyace en la "letra" de una serie de huaynos (waynu) quechuas de la sierra central y meridional del Perú.
\end{abstract}

Most recent works about Quechuas' (Central Andes) symbolical practices show that "cosmological expression" is not exclusive to one kind of performance discourse. Cosmological concepts are also included in those narrative discourses which reenact ritual practices. In this paper, I shall study the "poetic cosmology" implied in huaynos lyrics from the Meridional and Central Sierra Peruvian Quechuas. 
Acta Poetica 26 (1-2)

PRIMAVERA-OTOÑO

2005

Martin Lienhard

Universidad de Zurich

\section{La cosmología poética en los huaynos quechuas tradicionales ${ }^{1}$}

\section{Introducción}

Como lo han ido subrayando varios trabajos recientes sobre las prácticas simbólicas andinas, la expresión de lo "cosmológico" no es el privilegio exclusivo de ninguno de los numerosos discursos (paralelos, superpuestos o imbricados) que componen, en los Andes centrales, una performance ritual y/o musical. En este sentido, se ha atribuido relevancia cosmológica al empleo de determinados instrumentos y voces, la calidad de los sonidos producidos, la melodía y el ritmo (códigos musicales); al despliegue de los actores en el espacio, la coreografía y la gestualidad, las máscaras, el vestuario y el decorado

\footnotetext{
${ }^{1}$ Una primera versión de este trabajo se presentó en el simposio Cosmología y música en los Andes (Berlín, 1-6 de junio de 1992), evento organizado por el Instituto Internacional de Música Comparada, el Instituto Iberoamericano y el Instituto Latinoamericano de la Universidad Libre, todos con sede en Berlín. Para su reelaboración me resultaron útiles varias de las ponencias presentadas en esa oportunidad (especialmente las de Max Peter Baumann, Henry Stobart y John Schechter), así como las observaciones que me hicieron Rosalía Martínez, Verónica Cereceda y Rodrigo Montoya. Isabel Asto de Damián cantó todos los huaynos de Isua (Lucanas, Ayacucho) que figuran aquí como materiales de estudio. A ella van, una vez más, mis agradecimientos más sinceros.
} 
(códigos escénicos); a la inscripción de la performance en el calendario ritual y su desarrollo en el tiempo (códigos temporales). Desde luego, las concepciones cosmológicas se incrustan, también, en el discurso narrativo (códigos dramáticos y verbales) que actualizan las prácticas rituales, los cantos, etc.

Trataré a continuación de develar la "cosmología poética" que subyace a la "letra" de una serie de huaynos (waynu) quechuas de la sierra central y meridional del Perú. Una investigación de este tipo de trabajo supone ciertas precauciones teóricas y metodológicas.

La "letra" —o el texto verbal - no es sino uno de los elementos que entran a formar parte de la performance de un canto como el huayno. Por un lado, un texto musical (melodía, ritmo), acompañándolo o haciendo las veces de contrapunto, le sirve de soporte. Ambos se realizan por medio de una o varias voces que pertenecen a personas condicionadas por su experiencia personal y colectiva en un contexto socio-cultural e histórico determinado. El canto surge en el marco de una puesta en escena que implica, además de la actuación de los personajes (cantantes / oyentes), la ocupación de un espacio y la inserción en el tiempo (astronómico, social, histórico).

Sin embargo, el texto verbal - por su autonomía relati$v a-$ se rige, hasta cierto punto, según unas normas que le son propias. Las estructuras del texto (verbal) producen un sentido "objetivo" - accesible al análisis_ que su puesta en escena, a menos de ser paródica, no llega a anular del todo. Este sentido objetivo no coincide siempre ni necesariamente con el que le atribuyen, en el momento de la performance, los ejecutantes o el público. En lo que sigue, perfectamente consciente de los límites de esta indagación, intentaré, pues, poner de relieve la cosmología objetivamente incrustada en los textos verbales de algunos cantos.

Tratándose de textos producidos y "recepcionados" en un régimen de oralidad, es evidente que no podemos considerar- 
los como fijos o definitivos. En cada performance, los textos latentes (en la memoria de sus protagonistas) pueden sufrir modificaciones mayores o menores, desde la substitución de palabras o frases, y pasando por un montaje diferente de las "estrofas", hasta la incorporación momentánea de contenidos radicalmente nuevos. Varían también, desde luego, la "puesta en voz" y la "puesta en escena" del canto. Para los fines limitados de esta investigación, tales cambios y variaciones, sin embargo, no resultan decisivos: la cosmología poética de los cantos analizados parece demostrar, en realidad, una estabilidad considerable.

\section{Materiales}

Nos basaremos en una serie de huaynos "tradicionales", oriundos del centro-sur de la sierra del Perú y cantados, actualmente, por ciertos sectores campesinos o sus descendientes urbanos "tradicionalistas". Echaremos mano también, para algunas demostraciones, de cantos de otro tipo. ${ }^{2}$ Cabe subrayar que los huaynos tradicionales coexisten, en el área mencionada (y en sus ramificaciones urbanas y costeñas), con otros de elaboración más reciente y de características eminentemente distintas (cf. Baquerizo 1993).

Contrariamente a los cantos vinculados estrictamente a las fechas del calendario ritual (católico, agrícola y ganadero) y a los ritos de pasaje, el huayno quechua de la sierra central y meridional del Perú surge en cualquier momento del año y en las circunstancias sociales más diversas. Por eso, sin duda, se lo llama, en algunas comunidades, llaqtay taki: el canto-de-mipueblo. De todos los géneros musicales del área, no es sólo el

\footnotetext{
${ }^{2}$ Para la transcripción de términos o sintagmas del quechua me apoyaré en el alfabeto (trivocálico) actualmente en uso en el Perú. En los textos transcritos por otros estudiosos se conserva la grafía original.
} 
más difundido, sino también el que demuestra, en su ejecución, la mayor autonomía. Esto no significa que, en una comunidad campesina, en los caminos y carreteras o en un barrio urbano, cualquier huayno surja en cualquier momento, sino que su relación con un momento concreto no obedece a una "ley" previsible.

En sus variantes más difundidas, cantado por los mistis (miembros del antiguo sector señorial), los mestizos y amplios sectores de origen serrano en las grandes ciudades serranas y costeñas, el huayno alcanzó, en el Perú, el estatus de canción representativa de la cultura "serrana". En este sentido, el huayno cantado llegó a ser, desde hace varios decenios, la canción andina más difundida y comercializada, lo que pudo influir en la escasez de estudios dedicados a su música o sus textos, a pesar de que los huaynos tradicionales son, sin duda alguna, una expresión poética de notable trascendencia.

\section{Principios de la "poesía quechua”}

Ha habido varios intentos para definir los principios básicos de la "poesía quechua" (generalmente, los principios poéticos que rigen los textos verbales de los cantos quechuas). En sus importantes trabajos sobre los textos poéticos que figuran en la Primer nueva corónica... de Guaman Poma de Ayala, Philippe Husson $(1985,1993)$ subraya, por un lado, el empleo de un lenguaje poético — gramática y léxico- distinto del lenguaje coloquial o cotidiano, y por otro, la presencia constante de lo que él califica de paralelismo semántico: "correspondencias, al nivel del sentido, entre palabras homólogas situadas en secuencias contiguas" (Husson 1993, 65).

El paralelismo semántico surge, de hecho, en el marco del paralelismo gramatical. ${ }^{3}$ Preferimos, al hablar de poesía canta-

\footnotetext{
${ }^{3} \mathrm{El}$ potencial valor poético de los paralelismos gramaticales fue puesto de relieve por Roman Jakobson en su trabajo "Poésie de la grammaire et grammaire de la poésie" (Jakobson 1984, 127-153).
} 
da, partir de este fenómeno propiamente "rítmico". En los textos de los huaynos, los paralelismos gramaticales producen una especie de macro-ritmo binario, basado a veces en la simple repetición ("bis") de un sintagma poético, pero más generalmente en la repetición, con variantes léxicas, de una estructura gramatical:

Tumanaykipaq/ mana tarispa/ chaypi waqanki

Upyanaykipaq/mana tarispa/ chaypi llakinki

No hallando qué tomar/ ahí mismo llorarás no hallando qué beber/ ahí mismo sufrirás 4

Las palabras substituidas o variables atraen, desde luego, la atención de quienes "siguen" el texto cantado. Partiendo de los materiales ofrecidos por Guaman Poma, Husson (1993) se sirve, para definir la relación semántica entre las palabras substituidas, de los conceptos de sinonimia, parasinonimia y pertenencia al mismo campo semántico:

a) Sinonimia: ипu / yaku (agua / agua: diferencia dialectal).

b) Parasinonimia: llulla / pallqu (mentira / engaño).

c) Pertenencia al mismo campo semántico: asi- / puklla(reir/jugar); lliklla / aqsu (manta / saya).

Comentando las fluctuaciones semánticas que producen estos paralelismos, Husson $(1985,362)$ sugiere que la destrucción de la pertinencia denotativa de la palabra y el efecto de ambigüedad consecutivo auspician una lectura "emocional" del texto poético. Sus conclusiones tienden a insinuar, igualmente, que las variaciones semánticas (y otros procedimientos característicos de los poemas quechuas transcritos por Guaman Poma) son un espacio para la "libertad" del poeta.

\footnotetext{
${ }^{4}$ Huayno de Isua, Lucanas, Ayacucho, cantado por Isabel Asto de Damián, Lima, marzo de 1988. Grabación, transcripción y traducción de M. Lienhard.
} 
El estudio del fenómeno del paralelismo sintáctico en los huaynos y otros cantos quechuas modernos puede llevar sin duda a diversas conclusiones. En primer lugar, en los huaynos quechuas analizados, los elementos variables tienden a constituirse en parejas estables. La libertad de elección del poeta resulta sumamente restringida: poco menos que sistemáticamente, una variable determinada arrastra, en la repetición del sintagma que la engloba, la manifestación de su "pareja" tradicional. Si por ejemplo, en una construcción que paraleliza dos sintagmas poéticos, el primero contiene, como variable, una forma derivada de waqa- (llorar), podemos suponer - $\mathrm{O}$ estar casi seguros de - que el segundo ofrecerá la misma forma derivada de llaki- (sufrir):

Urqukunapi tarukitas/ taytay irranti waqawanqa urqupi vikuñitas/ mamay irranti llakiwanqa.

Taruka de los cerros/ mi padre, errante, llorará por mí Vicuña de los cerros/ mi madre, errante, sufrirá por mí (véase el texto núm. 3 del apéndice)

Llaki- y waqa-, "parasinónimos", son lexemas extraídos de un campo semántico que abarca las expresiones del llanto y la tristeza. En el lenguaje poético de los huaynos estudiados, ellos forman una pareja prácticamente obligatoria.

Si el primer sintagma contiene, como variable, el lexema ripu- (irse), el sintagma paralelo le substituirá, sin duda, el lexema pasa- (irse). La única diferencia importante entre estos términos sinónimos estriba en su origen, respectivamente quechua y español:

\section{Chaymantaqa ripusaqsi/ chaymantaqa pasasaqsi}

Entonces me iré/ entonces me marcharé 5

\footnotetext{
${ }^{5}$ Cf. nota núm. 2.
} 
A urqu ('cerro') le suele seguir, en las construcciones paralelas, qasa ('abra'):

Wzzaqllay urquta chinkakuptiy/ ima taytallay llakiwanqa waqllay qasata chinkakuptiy/ ima taytallay llakiwanqa

Cuando me pierda por aquel cerro/ cómo sufrirá mi padre cuando me pierda por aquella abra/ cómo sufrirá mi padre (madre) (véase el texto núm. 3 del apéndice)

Contrariamente a las variables anteriores, urqu y qasa no son sinónimos ni parasinónimos. Se diría, más bien, que los dos conceptos se oponen: si el primero evoca la parte alta de una cordillera, el segundo remite a los lugares donde el filo de la cordillera se quiebra. Ambos son, sin embargo, parte constitutiva de la cordillera en su conjunto: elementos cuya relación es de oposición y complementariedad.

La relación de oposición y complementariedad es más evidente aún en otra pareja de variables: mama y tayta.

kayninta pasaspan/ taytallay chinkarqun

kayninta pasaspan/ mamallay chinkarqun

por aquí pasando/ se perdió mi padre

por aquí pasando/ se perdió mi madre

(véase el texto núm. 5 del apéndice)

Pareja de variables absolutamente central en un gran número de huaynos, tayta y mama representan las dos caras o mitades obligatorias y exclusivas de una célula bifronte, fundamento de la sociedad en su dimensión biológica (en quechua, el 'padre' y la 'madre' nunca se funden, contrariamente a lo que se observa en los principales idiomas europeos, en un concepto único y dominado por la función paterna: 'padres' en español, 'parents' en inglés y francés, 'Eltern' en alemán, etc.). 
La contribución respectiva de cada una de las dos "mitades" de esta célula para la reproducción de la sociedad humana aparece también como posible variable en las construcciones paralelas:

Waqllay qaqa chaupipis / taytallay churiyallawarqa waqllay monte chaupipis / mamallay wachallawarqa

En medio de aquel roquedal/ mi padre me engendró en medio de aquel monte/ mi madre me parió ${ }^{6}$

En la misma línea, los paralelismos tematizan, también, la oposición entre el hijo/ la hija del padre y el / la de la madre:

manas mamallaypas / wawallay niwanchu manas taytallaypas / churillay niwanchu

ni siquiera mi madre/ hijo mío (hija mía) me dice ni siquiera mi padre/ hijo mío (hija mía) me dice (véase el texto núm. 6 del apéndice)

Sinónimos desde una perspectiva europea, churi ('hijo / hija del hombre') y warmi ('hijo / hija de la mujer') remiten a la diferencia que existe entre las miradas opuestas y complementarias del hombre y de la mujer.

La pareja celeste inti / killa, cuya homología con la pareja terrestre tayta / mama es notoria, "reina" en otras tantas construcciones paralelas:

Intipas yaykuykachkanña / killapas chinkaykuchkanña

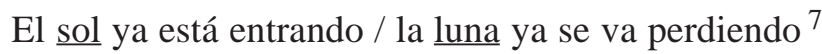

La pareja punchaw (día) / tuta (noche), bien documentada en los huaynos, no es mucho más que una variante de la pre-

${ }^{6}$ Cf. nota núm. 2.

${ }^{7}$ Cf. nota núm. 2. 
cedente: si punchaw es la mitad "solar" del día astronómico, tuta figura la otra mitad, cuya oscuridad se ve matizada por la presencia de la luna.

Quri y qullqi (oro/ plata) configuran otra pareja típica. Si bien la serie léxica de los metales autorizaría, teóricamente, otras parejas, ésta es la que se impone en el lenguaje poético. La sintaxis del ejemplo siguiente insinúa, sin lugar a dudas, la homología de la relación madre / padre y oro / plata:

Yuyarillaway mamay / yuyarillaway taytay

qori kiraupi uywallawasqaykita / qollqi kiraupi uywallawasqaykita

Acuérdate madre mía/ acuérdate padre mío

yo, criado por ti en una cuna de oro / yo, criado por ti en una cuna de plata

(véase el texto núm. 2 del apéndice)

Parece, en resumen, que la substitución de lexemas en las construcciones paralelas se apoya, por un lado, en la existencia de unas parejas de sinónimos consolidadas por y dentro de la tradición poética, y por otro, en la de las parejas previstas, independientemente del discurso poético, en la "lengua” —en el sentido de Saussure - y la cultura quechua.

Varias de estas parejas se pueden considerar como las coordenadas de una cosmología: sol / luna, día / noche, oro / plata, padre / madre, cerro / abra, etc. Como tales, algunas de ellas tienen, en la "poesía" quechua, una larga tradición. El himno a Wiraqocha que transcribió, a comienzos del siglo XVII, el cronista Pachacuti Yamqui Salcamaygua (texto núm. 1 del apéndice), presenta, entre otras, las parejas inti / killa (sol / luna) y punchaw / tuta (día / noche). En vez de la pareja tayta / mama encontramos, en este texto antiguo, la de qari y warmi (hombre / mujer). En todas ellas "vibra", sin lugar a dudas, la oposición cosmológica "primordial" hanan/ urin (arriba / abajo). 
Podemos constatar que el texto transcrito por Pachacuti Yamqui se sirve de paralelismos gramaticales - muy simples- para manifestar una serie de oposiciones cosmológicas básicas:

\section{Intiqa killaqa/ p'unchawqa tutaqa / puquyqa chirawqa}

Y el sol y la luna/ y el día y la noche / y el verano y el invierno

Si el análisis puramente gramatical de esta serie sugiere la sucesión de seis elementos idénticos (nombre, sufijo -qa), la introducción de criterios semánticos revela, atribuyendo el calificativo cosmológico hanan (arriba) a los nombres impares, y el de urin (abajo) a los pares, un ritmo de oscilación pendular. En los cantos modernos, la multiplicación de las parejas de oposición suele adoptar formas dispositivas menos canónicas. Si volvemos a leer el fragmento de canto de velorio apenas citado, constatamos que la primera pareja, mama / tayta, invierte el orden hanan / urin que manifiesta la segunda, quri / qullqi. Dicho de otro modo, si la calidad de la oposición es la misma en ambos casos, su ordenamiento es otro. Como quiera que sea, las oposiciones de tipo cosmológico, lejos de estar simplemente presentes en el discurso poético, aparecen como el principio básico de su composición. Al crear, gracias al sistema de la substitución léxica, un ritmo de oscilación pendular, los paralelismos sintácticos sugieren la imagen de un cosmos dominado por oposiciones complementarias. Ritmo pendular y dualismo conceptual se refuerzan mutuamente.

¿En qué medida todas las parejas léxicas del paralelismo sintáctico aquí ejemplificadas se ajustan, aun cuando no son de directa relevancia cosmológica, a ese sistema cosmológico dual? En los materiales presentados aparecen, fuera de las parejas ya mencionadas, otras más. En el canto de velorio (texto núm. 2 del apéndice), el padrino y la madrina, donadores res- 
pectivamente de la vestimenta y del arado, no constituyen sino una ampliación de la pareja de oposiciones tayta / mama.

kuyay madrinaypa pachachiwasqanwan

kuyay padrinuypa taclla quwasqanwan

con el vestido que mi querida madrina me regaló

con el arado que mi querido padrino me regaló

En uno de los cantos (texto núm. 4 del apéndice) se forma la pareja rumi y sacha (piedra / árbol) paralelamente a la oposición tayta / mama. En otro texto, transcrito en su totalidad en el apartado siguiente de este artículo, la pareja qaqa (roca) y munti (monte) aparece siempre en el contexto de una oposición tayta / mama. En otro todavía (texto núm. 6 del apéndice), el texto desarrolla un paralelismo sintáctico que abarca tres parejas asociadas: abajo / arriba (del camino), pukuy / liwli (sendas avecitas), madre / hermana (del hombre):

Nanpa uranmanta/ pukuy pukuychalla, ñanpa hawanmanta/ liwli liwlichalla, panaymanta, mamaymanta tukuyuspalla...

debajo del camino/ sólo el pukuy pukucha encima del camino/ sólo el liwli (liwlicha) en mi hermana/ en mi madre convertidos

En los cantos núm. 3 (huayno) y núm. 4 (santiago: canto de la marcación del ganado) se paralelizan la taruka y la vicuña. En el primer caso, taruka y vicuña remiten respectivamente a tayta y a mama,

Urqukunapi tarukitas / taytay irranti waqawanqa urqupi vikuñitas / mamay irranti llakiwanqa

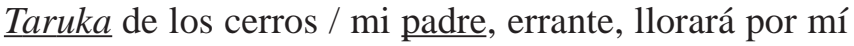
Vicuña de los cerros / mi madre, errante, sufrirá por mí 
mientras que en el segundo, la relación entre ambas parejas no es paradigmática, sino puramente sintagmática:

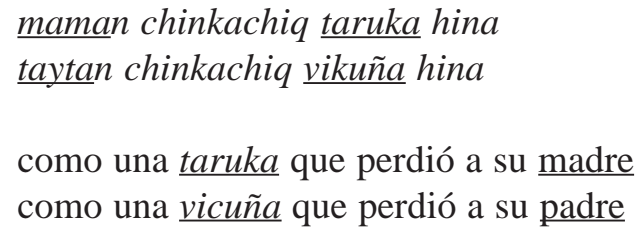

En el canto santiago apenas mencionado aparece, todavía, la pareja para / lasta (lluvia / nieve); en el huayno núm. 5, la de aycha y yawar (carne / sangre). En otro huayno, cuya transcripción completa se halla en el apartado siguiente, se vincula, en cambio, sendos conceptos de origen español: destino y pecado:

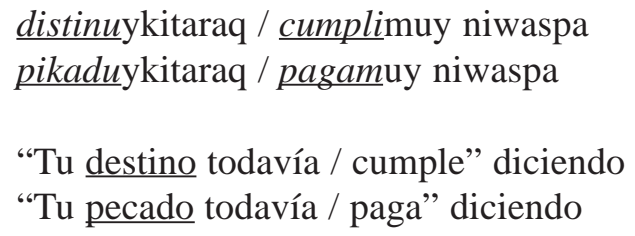

Algunas de estas parejas se podrían considerar, quizás, como variantes menores de la oposición cosmológica primordial hanan / urin: sacha (árbol) y rumi (piedra), en efecto, pueden hacer pensar en una oposición masculino / femenino, mientras que qaqa (roca) y munti (monte, selva) recuerdan la oposición tierras altas / tierras bajas). Pero ¿cómo explicaríamos, a partir de esta óptica, que dos conceptos muy parecidos, rumi (piedra) y qaqa (roca), asuman posiciones opuestas?: rumi se asocia a mamay, mientras que qaqa se relaciona con taytallay. Sin duda, la solución de este problema no se halla en una supuesta "esencia" distinta de uno y otro elemento.

En cuanto a las parejas de sinónimos compuestos por un lexema quechua y otro español (ripu-, pasa-), sugiere la presen- 
cia de una división cosmológica (mundo quechua / mundo español), pero ¿en qué medida los hablantes tienen conciencia del origen divergente de estas palabras? ¿Y qué connotaciones cosmológicas se podrían atribuir a pecado y destino, o a las dos manifestaciones tópicas de la lamentación, llaki- y waqa-? La "bifurcación", en estos casos, sugiere más bien que cada ser, objeto o práctica ofrece dos vertientes, a veces - como en la oposición wawa y churi- producto de dos miradas opuestas y complementarias. En muchas construcciones paralelas, la dimensión cosmológica (básicamente, la oposición arriba / abajo) se cifra menos en la calidad de los conceptos opuestos que en la propia forma binaria.

Propondremos, pues, la hipótesis siguiente: en el discurso poético, los paralelismos construidos sobre la base de alguna de las oposiciones cosmológicas primordiales arrastran otras oposiciones que se tiñen, contextualmente, del "color" de la oposición principal. Si algunas de las variables sin significación cosmológica precisa pueden al menos connotarlas, otras, en cambio, sólo las adquieren en el contexto poético.

\section{“Cosmología poética” en movimiento}

Los cantos examinados no se limitan a construir una cosmología poética estática, a través de sus paralelismos sintácticos y la constitución de parejas. A partir de la perspectiva de un yo determinado, cada uno narra una historia o presenta una sucesión de imágenes. La cosmología no aparece sola, sino en relación con una mirada y, posiblemente, una "acción". Esto se puede observar en el texto verbal del siguiente huayno:

Waqllay qaqa chaupipis

taytallay churiyallawarqa

waqllay munti chaupipis 
mamallay wachallawarqa

liunpas tigripas

tragarquchun nispa

tigripas liunpas

mikurquchun nispa

Tigricha liuncha llaqa manallas mikullawanchu

tigricha liuncha llaqa

manas tragallawanchu

distinuykitaraq

cumplimuy niwaspa

pikaduykitaraq

pagamuy niwaspa

ima ñuqallay pubri

distinuyta cumplichkayman

ima ñuqallay pubri

pikaduyta pagachkayman

mamallaytaraqchu

ñuqa waqachirqani

taytallaytaraqchu

ñuqa waqachirqani

En medio de aquel roquedal

mi padre me engendró

en medio de aquel monte

mi madre me parió

"león y tigre

lo (la) traguen" diciendo

"tigre y león

lo (la) devoren" diciendo.

Tigre y león

comerme no quisieron

Tigre y león

tragarme no quisieron

"Tu destino todavía 


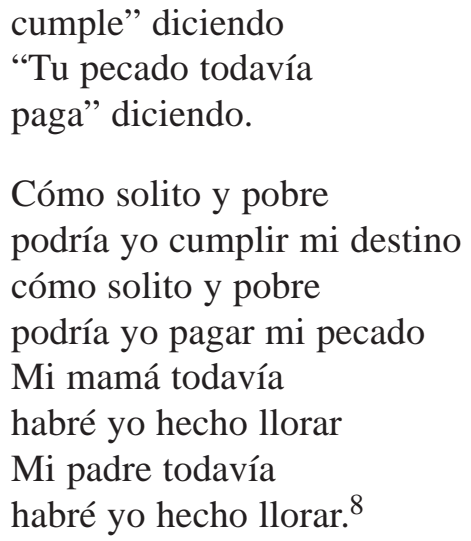

Si muchos huaynos toman, desde el comienzo, la forma de una invocación a un elemento de la naturaleza (generalmente una planta, un animal, una piedra), el huayno apenas transcrito pertenece, en cuanto a su forma de enunciación aparente, al género narrativo. La primera y la segunda estrofa "narran", en efecto, el origen y la persistencia de la soledad que afecta al yo, al hablante poético. Con la tercera estrofa, sin embargo, el discurso cambia de signo. Sin lugar a dudas, Ima ñuqallay pubri significa una invocación, dirigida a una instancia no nombrada. ¿Cuál podría ser tal instancia? Al parecer se trata de un poder superior no nombrable, situado por encima de la pareja padre-madre y por encima de la pareja tigre-león. Si la pareja padre-madre remite a la sociedad de los hombres, la pareja tigre-león parece evocar las fuerzas, no controladas por el hombre, de la naturaleza salvaje. Abandonado por la sociedad y colocado ante sus "responsabilidades" por los supuestos agentes de la naturaleza incontrolada, el yo poético invoca, pues, a quien domina a ambas.

Los textos verbales de muchos waynu campesinos se centran en la relación entre una pareja padre / madre y el yo poé-

\footnotetext{
${ }^{8}$ Cf. nota núm. 2. Nótese que en una primera versión, interrumpida casi enseguida por no darle satisfacción, la cantante - Isabel Asto- había introducido, como variables, qasa y urqu
} 
tico. La ruptura de esa relación constituye, por lo común, su tema principal. A menudo, un suceso más o menos concreto - fracaso amoroso, prisión (texto núm. 6), servicio militarobliga al yo a abandonar la protección que le solía brindar la pareja padre / madre. Otras veces, las causas de la ruptura resultan más difusas. En Viseca wayqupi (texto núm. 5 del apéndice), el yo poético acusa al pejerrey, habitante del río que corre en la quebrada, de haber devorado a su padre y su madre (según una explicación corriente, los huaynos de este tipo se refieren a accidentes como el desbarrancamiento de un camión con sus pasajeros, pero el texto permite también otras interpretaciones).

En nuestro huayno, quien provoca la ruptura es la propia pareja padre / madre. Hasta donde sabemos, se trata de una figura mucho menos corriente que las anteriores. Si tomamos al pie de la letra el argumento del huayno, se evoca el abandono del hijo (o de la hija) por parte de su padre y su madre carnales. ¿Ilustración de una tragedia concreta? Nada permite afirmarlo. El texto carece, en todos sus niveles, de referencias a personajes o hechos concretos. A todas luces, la pareja padre / madre no remite nunca, en la poética de los huaynos, a personas concretas. Creemos que su función es otra. En los poemas prehispánicos (donde no parece existir —o no llamar la atención- esa pareja), el hablante poético podía solicitar, para enfrentar su angustia, la protección divina. El hombre andino colonial o moderno perdió, con la conquista y la desestructuración de su sociedad, ese recurso supremo. En los poemas poshispánicos, la pareja padre-madre toma, de algún modo, el lugar de esa instancia central desaparecida. En los huaynos tradicionales, ella funciona, en efecto, como una nueva instancia de protección. Los paralelismos sintácticos enfatizan la índole de "célula única de dos caras" que significa, en los textos, la pareja padre / madre. Contrariamente a lo que sucede - y con la mayor frecuenciaen la realidad social, el padre o la madre no suelen, en los huaynos, aparecer "sueltos". Los huaynos tradicionales están lejos, 
en rigor, de querer "retratar" situaciones sociales concretas. En un huayno apurimeño (texto núm. 6 del apéndice), el texto pone en paralelo - fuera de cualquier norma conocida - a una madre y a una hermana. Quizás, en este caso, el autor (un abigeo con experiencia de cárcel), se refiera, efectivamente, a una situación concreta. En el mismo texto, sin embargo, aparece también, tributo a la tradición poética con su cosmología estable, la pareja tayta / mama.

Pensamos que la pareja padre / madre representa, ante todo, a la colectividad humana, a la "comunidad". Desde la destrucción del estado autóctono y de sus diferentes mecanismos de encuadramiento y de protección, la comunidad resulta, en efecto, la "familia" que proporciona una seguridad mínima a sus "hijos". En los huaynos tradicionales, el hombre andino se piensa, en efecto, en términos de "hijo" — de la comunidad-. No aparece nunca como individuo autónomo y autosuficiente. Cuando surge, el estatus de "individuo" aparece como resultado de una pérdida o ruptura, no como opción social. Producto de una situación adversa, el "individuo" no es sino un huérfano, un waqcha.

En buena medida, los huaynos se dedican a mostrar a un hijo amenazado de orfandad o a un huérfano soñando con la recuperación de su estatus de hijo. Su tema fundamental sería, por lo tanto, la "crisis" — una crisis duradera - en la relación entre el hombre y la sociedad (y el cosmos). Lo que anhela el yo poético es el restablecimiento de esta relación.

En el huayno que estamos estudiando, la desestructuración del mundo aparece como un hecho indiscutible. La orfandad del yo no es una amenaza, sino una triste realidad presente. No un suceso, sino un elemento "estructural". Desde el comienzo, la pareja padre / madre se niega a asumir su función cósmicosocial: la de garantizar la reproducción y la protección de la sociedad humana. ¿Cómo explicar este rechazo? Para la sociedad andina, los hijos representan un "capital" decisivo. Su 
abandono o rechazo no es, pues, una práctica corriente. El abandono evocado aquí remite, entonces, a una situación que impide la reproducción normal de la sociedad y la empuja al suicidio. ¿En qué momento de la historia andina habrá surgido el tema de este huayno? ¿En la época de la conquista, en la de la derrota de Túpac Amaru (fines del siglo XVIII), o en la de la expansión latifundista (siglo XIX)? No conocemos la respuesta, pero la existencia actual de este huayno implica su vigencia colectiva. La memoria colectiva de una comunidad no conserva, en efecto, temas, motivos o formas que han perdido toda su vigencia.

El abandono del hijo por la pareja padre / madre es sólo la primera etapa del argumento. La segunda parte introduce otro rechazo de signo diferente: las fieras león y tigre se niegan a devorar a la víctima que se les ofrece tan explícitamente. ¿Por qué? ¿Quiénes son los personajes que nombran estos dos lexemas de origen español? Si liun remite a puma, felino predador conocido en las tierras altas, tigri equivale al uturunqu o jaguar, felino que representaba, en la cosmología incaica, al Antisuyu, la zona alto-amazónica: el cuadrante dominante de la mitad de abajo urin. Ellos encarnarían, pues, el inquietante y mal conocido subuniverso urin. A pesar de su sorprendente mansedumbre, el tigre y el león no son realmente, como se podría pensar en un primer momento, protectores del yo poético. Tampoco son exactamente representantes de un mundo "salvaje". A todas luces, ellos defienden o imponen una especie de orden. Este orden exige que el hombre asuma sus "responsabilidades". Ahora, los conceptos que sirven para nombrar estas responsabilidades son, como sus propios nombres, de origen español y judeo-cristiano: "cumplir su destino" y "pagar sus pecados". Si la noción del pecado remite, de por sí, al pensamiento cristiano, la narración puntualiza que se trata, muy concretamente, del "pecado original", del pecado de quienes no tuvieron (todavía) la posibilidad de cometerlo. La obligación de 
"cumplir su destino" equivale a la prohibición del suicidio, otro principio de marca judeo-cristiana. Los dos felinos "salvajes" resultan, pues, dos abogados del orden cristiano: un orden relegado, dentro de la cosmovisión del poema, al subuniverso urin, a la "no cultura". La representación de los agentes del sistema colonial bajo el aspecto de fieras, especialmente de felinos, parece corresponder a cierta tradición andina. ${ }^{9}$

Abandonado por la pareja padre / madre y "rechazado" también por las fieras, el yo poético se halla como expulsado del (de su) mundo y expuesto a la soledad y al desarraigo más completos y trágicos. No se trata, ciertamente, de una soledad y un desarraigo individuales. El suceso que aquí se narra no lleva, en efecto, rasgos de anécdota concreta e individual. Igual que las parejas padre / madre y león / tigre, el yo no remite a ninguna categoría individual (ni sexual): es un yo plural.

En varias oportunidades, el narrador, poeta quechua y antropólogo José María Arguedas aludió a la "soledad cósmica" que manifiesta el pueblo quechua en sus cantos. Él la atribuía al resentimiento de un pueblo "vencido" (1961). Sin duda, la poesía prehispánica conocía cierto tipo de "soledad cósmica". Basta recordar, a este propósito, la pregunta obsesiva del yo poético de los himnos prehispánicos por el lugar de residencia de la divinidad: Maypim kanki? (‘¿Dónde estás?’, cf. texto núm. 1 del apéndice). Pero aquí, como en otros cantos recogidos a lo largo del siglo xx, la soledad aparece como más definitiva y radical. No hay siquiera a quien comunicarla, a quien solicitar protección. Hemos visto que la "divinidad" a quien invoca la última estrofa del huayno no lleva nombre. En el siglo xx no se puede ya invocar a un Wiraqucha remoto en el tiempo y el espacio, "confundido" además con los conquistadores y sus descendientes o sucesores (los wiraquchas). Pero tampoco tiene

\footnotetext{
${ }^{9}$ En un dibujo alegórico de Guaman Poma (1980, 694-695/708-709) que representa a los diversos enemigos de los indios en el sistema colonial, el tigre y el león simbolizan, respectivamente, a los "españoles del tambo" y al "(en)comendero".
} 
sentido invocar al Dios cristiano, divinidad de los opresores y cúspide de una religión que subraya el "pecado original", un pecado que pesa sobre los inocentes y los débiles. Ante este gran vacío, se invoca, pues, a una autoridad sin nombre ni residencia conocida.

Observada en sus elementos "fijos" y en su movimiento, la "cosmología poética" que manifiesta el texto verbal de este y de muchos otros huaynos, diseña un mundo en crisis, cuyos rasgos remiten a la desestructuración que sufrió la sociedad andina a raíz de la conquista. El hecho de que tales textos se sigan memorizando, reproduciendo y desarrollando, indica la vigencia del sentimiento expresado, que va alimentada por todos los factores nuevos de desestabilización que se vienen agregando a los "viejos".

El "sentido objetivo" que se desprende del texto verbal de los cantos (v. introducción) no coincide necesariamente con el sentido que se desprende de los textos no verbales ni con el que le atribuirían, en el momento de su performance, sus ejecutantes y su público. La captación de ese otro sentido "variable" exigirá, naturalmente, la elaboración de otro enfoque: tarea que se reservará a futuras investigaciones.

\section{Apéndices}

\section{Pachacuti Yamqui Salcamaygua}

(Reconstrucción y traducción: J. Szeminski 1993, 131-132)
¡A Wira Quchan, tiqsi qapaq, "kay qari kachun", "kay warmi kachun" 
ñiq apu,

hinantin achikcha kamaq!

¿Maypim kanki?

¿Manachu rikuykiman?

¿Hananpichum,

hurimpichum,

kinrayñinpichum

qapaq usñuyki?

Intiqa killaqa

p'unchawqa tutaqa

puquyqa chirawqa

manam yanqachu.

¡O Almácigo de sustancia vital,

rey que pone fundamentos,

"éste sea varón",

"ésta sea mujer",

señor que lo dice,

alma que crea la primera luz de todas las cosas.

¿Dónde estás?

¿No podré verte?

¿En el lugar de arriba,

en el lugar de abajo,

en la tierra

está tu real usnu?

El sol y la luna,

el día y la noche,

el verano y el invierno

no son en vano.

\section{Wawa pampay (qarawi)}

Huamanquiquia (Víctor Fajardo, Ayacucho) 
Recopilación: Alina Cavero, en Jesús Armando Cavero 1985, 246. Traducción de M. Lienhard.

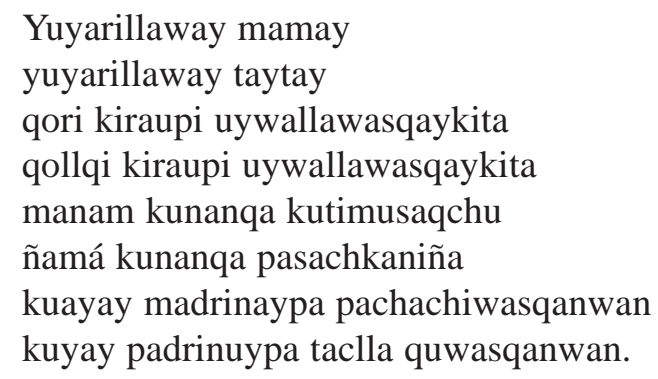

Acuérdate madre mía acuérdate padre mío yo, criado por ti en una cuna de oro yo, criado por ti en una cuna de plata no volveré más ahora ya me estoy yendo ahora con el vestido que mi querida madrina me regaló con el arado que mi querido padrino me regaló.

\section{Wamanchallay killinchallay}

Huayno de Isua, Lucanas, Ayacucho, cantado por Isabel Asto de Damián, Lima, marzo de 1988. Grabación, transcripción y traducción de M. Lienhard.

Waqllay urquta chinkaykuptiy ima taytallay llakiwanqa waqllay qasata chinkaykuptiy ima taytallay llakiwanqa (¿mamallay?)

Urqukunapi tarukitas

taytay irranti waqawanqa

urqupi vikuñitas

mamay irranti llakiwanqa. 
Cuando me pierda por aquel cerro

cómo sufrirá mi padre

cuando me pierda por aquella abra

cómo sufrirá mi padre (madre)

Taruka de los cerros

mi padre, errante, llorará por mí

Vicuña de los cerros

mi madre, errante, sufrirá por mí.

\section{Orqokunapa ñoqa yachani}

Santiago (canto de la marcación del ganado) de la sierra central del Perú, tomado de Sergio Quijada Jara 1957, 250. Traducción de M. Lienhard

Orqokunapi ñoqa yachani qasakunapi ñoqa yachani maman chinkachiq taruka hina taytan chinkachiq vikuña hina.

Wakllay taytallay nispa niptiyqa wakllay mamallay nispa niptiyqa hatun rumitaq mamay tukusqa hatun sachataq taytay tukusqa

Orqokunapi wayllay ichuchapas qasakunapi wayllay ichuchapas para chayaptin kurkuykachansi lasta chayaptin kumuykachansi

Chaynam ñoqapas kumuykachani chaynam ñoqapas usuykachani sutichallayta runa rimaptin sutichallayta runa parlaptin

Entre cerros yo vivo entre abras yo vivo 
como una taruka que perdió a su madre

como una vicuña que perdió a su padre.

Cuando dije allá está mi padre cuando dije allá está mi madre mi madre, una piedra grande había sido mi padre, un árbol grande había sido

En los cerros el ichu lozano en las abras el ichu lozano cuando llega la lluvia se agacha cuando llega la nieve se curva.

Así también yo me curvo así también yo me humillo cuando la gente habla mi nombre cuando la gente conversa mi nombre.

\section{Viseca wayqupi}

Canto de Puquio, Lucanas, Ayacucho, gentilmente ofrecido por Carlos Gutiérrez. Transcripción y traducción de M. Lienhard.

\footnotetext{
Visekay wayqupi

Piqiriyischallay (bis)

kayninta pasaspan

taytallay chinkarqun

kayninta pasasqan

mamallay chinkarqun

Lwuigucha lwigucha taytayta mikurqunki lwigucha lwigucha mamayta mikurqunki chayqayá kiruyki aycha aychallanña
} 
chayqayá simiyki

yawar yawarllanña.

De la quebrada de Viseca

mi pejerrey (bis)

por aquí pasando

se perdió mi padre

por aquí pasando

se perdió mi madre.

Lueguito luego

devoraste a mi padre

lueguito luego

devoraste a mi madre

mira que tus dientes

de carne están llenos

mira que tu boca

de sangre está llena.

6. Preso

Huayno de la comunidad de Wirapanpa, tomado de Ricardo Valderrama Fernández y Carmen Escalante Gutiérrez (1993, 30).

Panputa panpachata

prisuta rishaqtiy

Trapichi q'asata prisucha rishaqtiy, manas mamallaypas wawallay niwanchu manas taytallaypas churillay niwanchu. Ñanpa uranmanta pukuy pukuychalla, ñanpa hawanmanta liwli liwlichalla, 
panaymanta, mamaymanta

tukuyuspalla, rimaykamuwanpas tapuykamuwanpas Ñallas pasaniña.

Cuando iba presito por la pampita de Pamputa cuando iba presito por la abra de Trapichi ni siquiera mi madre hijo mío me dice ni siquiera mi padre hijo mío me dice debajo del camino sólo el pukuy pukucha de encima del camino sólo el liwli (liwlicha) en mi hermana en mi madre convertidos, siquiera me hablan por lo menos me preguntan Ya me estoy yendo.

\section{REFERENCIAS}

Arguedas, José María, 1961. "La soledad cósmica en la poesía quechua", Idea, artes y letras año XII, 48-49, julio-dic., 1-2.

BAQUERIZO, Manuel J., 1993. "La transición de la visión india a la visión mestiza en la poesía quechua oral", Revista de Crítica Literaria Latinoamericana 37-1 (Lima / Pittsburgh), 117-124.

Cavero, Jesús Armando, 1985. "El qarawi y su función social", Allpanchis 25 (Cusco), 233-270. 
Husson, Jean-Philippe, 1985. La poésie quechua dans la chronique de Felipe Waman Puma de Ayala, Paris, L'Harmattan.

—, 1993. "La poesía quechua prehispánica. Sus reglas, sus categorías, sus temas, a través de los poemas transcritos por Waman Puma de Ayala", Revista de Crítica Literaria Latinoamericana 37-1 (Lima / Pittsburgh), 63-85.

JAKOBSON, Roman, 1984. Une vie dans le langage - Autoportrait d'un savant, Paris, Minuit.

LIENHARD, Martín (ed.), 1993. "Aproximaciones a las prácticas textuales quechuas", Revista de Crítica Literaria Latinoamericana 37-1 (Lima/ Pittsburgh), 5-301.

Poma de Ayala, Felipe Guaman, 1980. Primer nueva coronica y buen gobierno, edición crítica por John Murra y Rolena Adorno, México, Siglo XXI.

QuiJada JARA, Sergio, 1957. Canciones del ganado y pastores, Huancayo, Talleres gráficos Villanueva.

SzEminski, Jan, 1993. "Manku Qhapaq Inka ¿un poeta religioso?", Revista de crítica literaria latinoamericana 37-1 (Lima / Pittsburgh), 131-158.

Valderrama Fernández, Ricardo y Carmen Escalante Gutiérrez, (eds.), 1993. "Canciones de imploración y de amor en los Andes. Literatura oral de los quechuas del siglo xx", Revista de Crítica Literaria Latinoamericana 37-1 (Lima/Pittsburgh), 11-39. 